\title{
Towards reducing misrepresentation of national achievements in marine protected area targets
}

Patrick Smallhorn-West ${ }^{a, b}$, Hugh Govan ${ }^{c}$

a ARC Centre of Excellence for Coral Reef Studies, James Cook University, Townsville, QLD 4811, Australia

${ }^{b}$ School of Earth and Environmental Sciences, James Cook University, Townsville, QLD 4811, Australia

${ }^{c}$ Adjunct Senior Fellow, University of the South Pacific (USP), School of Government, Development \& International, Affairs (SGDIA), Suva, Fiji

This is the accepted manuscript of a paper originally published in Marine Policy. You may find the final, formatted version at 10.1016/j.marpol.2018.05.031.

\section{Abstract}

Parties to the Convention on Biological Diversity (CBD) adopted 20 targets, known as the Aichi Targets, to benchmark progress towards protecting biodiversity. These targets include Target 11 relating to Marine Protected Area coverage and the World Database on Protected Areas (WDPA) is the accepted international database for tracking national commitments to this target. However, measuring national progress towards conservation targets relies on sound data. This paper highlights the large-scale misrepresentation, by up to two orders of magnitude, of national marine protected area coverage from two Pacific Island nations in multiple online databases and subsequent reports, including conclusions regarding achievements of Aichi 11 commitments. It recommends that for the target driven approach to have value, users of the WDPA data should carefully consider its caveats before using their raw data and that countries should strive for a greater degree of accountability. Lastly it also concludes that protected area coverage may not be the best approach to environmental sustainability and that the remaining 19 targets should be considered to a greater extent.

\section{Introduction}

With global declines in marine biodiversity there is a strong need for reliable international commitments for the sustainable management of the oceans. The highest level of international commitment is embodied by the Strategic Plan for Biodiversity 2011-2020 and its 20 Aichi Targets, which were adopted by the parties to the Convention on Biological Diversity (CBD) [1]. The expansion of protected area coverage is Aichi target 11, which by 2020 calls for 10 per cent of coastal and marine areas to be effectively and equitably conserved through protected areas and other effective area-based conservation measures. In this article we define MPAs using the CBD definition of "any defined area within or adjacent to the marine environment, together with its overlying waters and associated flora, fauna and historical and cultural features, which 
has been reserved by legislation or other effective means, including custom, with the effect that its marine and/or coastal biodiversity enjoys a higher level of protection that is surroundings" [2].

The Aichi 11 target of $10 \%$ protection for marine and coastal areas by 2020 has received much global attention. While the relation between coverage and conservation impact is disputed [3], it should be a relatively easy to measure indicator by which to assess the commitment of countries to marine conservation. National contributions to this target are often measured using the World Database on Protected Areas (WDPA) (www.protectedplanet.net) as well as other similar online datasets (e.g. MPA global - www.mpaglobal.org, PIPAP - www.pipap. sprep.org and Reefbase - www.reefbase.org). Combined, these databases form the backbone of reporting on progress towards meeting Aichi 11 and other global marine conservation targets. The criteria used in the WDPA for calculating protected area coverage are based on the IUCN and CBD definitions of protected areas. Changes to protected area coverage can be submitted to the WDPA only by government agencies; updates by external bodies (e.g. NGOs) are recommended to be submitted jointly with the management authority of each country [4].

National progress towards global conservation targets can only be assessed when the data employed are sound. Given the widespread use of the online datasets in measuring MPA coverage [5-8] it is important to ensure that they accurately measure the progress of nations towards stated targets. With large datasets some error is inevitable and misrepresentations of MPA coverage can arise from the existence of paper parks [9], protected area downgrading, downsizing and degazettment [10] or mistakes in the database [11]. However, while minor errors in online datasets could be judged as inevitable, gross and persistent misrepresentations of national achievements can undermine the overall process of marine conservation and should be addressed as a priority.

Misrepresentations in protected area coverage can result from both over- and underrepresentation of actual targets. While Visconti et al. [11] discussed the ramifications of underrepresentation due to limitations in the WDPA for identifying small-scale and community-based protected areas, large-scale overestimations of protected area coverage have received less attention. The consequences of overestimates in protected area coverage can be particularly negative as they may also lead to the subsequent cessation of conservation action if targets appear to have been met. Flow on effects, whereby management authorities aim to replicate reported success stories and researchers aim to understand factors leading to success are also more likely to arise from over, rather than under-representation of protected area coverage.

Attentive observers of the impressive and blossoming MPA coverage in the Pacific (e.g. Marae Moana - Cook Islands; Le Parc Naturel de la Mer de Corail - New Caledonia) may have noticed strange oscillations in the total figure and achievement of national targets [12]. Investigating misrepresentation of protected area coverage in this region is particularly pertinent given recent high-profile commitments and increasing claims of moral high ground in ocean stewardship. This paper uses two case studies from Tonga and Kiribati to draw attention to the large-scale misrepresentation, by up to two orders of magnitude, of national MPA coverage in 
this region, arising from both the long-term persistence of a massive paper park and data/quality control errors in the WDPA reporting process. Both large-scale misrepresentations occur in multiple online databases and subsequent reports, including conclusions regarding achievements of Aichi 11 commitments. It concludes that countries should actively strive for a greater degree of external accountability and that users of the WDPA should carefully consider its caveats before using their raw data for the target driven approach to have value.

\section{Tonga}

Tonga's marine protected area coverage on the WDPA (as of 31/01/2018) is reported as $10,133.87 \mathrm{~km}^{2}$, from 17 MPAs. Of these by far the largest is the Ha'apai Conservation Area (HCA), endorsed in 1995 by the Ha'apai Development Committee and Cabinet as a 10,000 km² multi-use conservation area in central island group of Tonga, at a cost of \$179,000 USD [13]. However, there is no evidence that the HCA, which is listed in the WDPA, PIPAP, MPA global and Reefbase, has enjoyed a higher level of protection than its surroundings in the last fifteen years. This conclusion is directly corroborated by several sources. Two reports on the current status of MPAs in the South Pacific [14,15] both deemed the HCA as inactive and recommended its removal from the national list of MPAs and therefore the WDPA. As early as 2002 the terminal evaluation of the South Pacific Biodiversity Conservation Program (SPBCP) also suggested that the HCA was unworkable due to a disconnect between the community, who saw the HCA as a government project, and the coordinating committee of government employees who did not feel ownership for the area. Two consultants, a government official and community members were also asked about the current status of the HCA and stated either that it is not currently active or that it was not achieving any more management than adjacent areas. One consultant expanded on this to confirm that there is no information on the legal status or management of the HCA, and no management plan has been found during several local reviews by Ministry officials.

The WDPA additionally lists 16 other MPAs in Tonga, nine of which were established in the 1970's and collectively cover $34.65 \mathrm{~km}^{2}$ and six of which were established as part of the Special Area Management (SMA) program starting in 2006 and cover $99.22 \mathrm{~km}^{2}$. The SMA program is currently under a rapid expansion and in 2017 implemented or was in the process of implementing an additional 18 areas and a further 25 on a waiting list. The best estimate of the current of the coverage of discussion with consultants and community members support the conclusion that lack of local acceptance and limited resources for enforcement resulted in management for the non-SMA areas becoming non-existent shortly after their inception.

In conclusion it is apparent that both by area and by number Tonga's MPA coverage has been over-reported. The reported figures are $10,133.87 \mathrm{~km}^{2}$ from 17 marine parks, whereas the realistic current figure of those listed on the WDPA is $99.22 \mathrm{~km}^{2}$ from seven active marine parks. This represents a two order of magnitude discrepancy between the WDPA database and the actual figures. In Govan [14] the coverage of the HCA was sufficient to account for approximately $40 \%$ of total MPA coverage in the South Pacific (as of 2009). 


\section{Kiribati}

The Phoenix Island Protected Area (PIPA) in Kiribati was one of the largest marine protected areas in the world when established in 2006, and according to the WDPA (31/01/2018) its representative marine area is $397,447 \mathrm{~km}^{2}$. In 2010 PIPA was also inscribed as a World Heritage area and on inscription was added to the WDPA database, inadvertently leading to a double entry of its size. Govan [15] warned that at least as late as 2013 the WDPA dataset listed the total coverage of PIPA as $818,750 \mathrm{~km}^{2}$ owing to its double counting. This misrepresentation has now been partly corrected: the online WDPA portal lists the corrected figure, however the MPA is still listed twice in the raw database, with the representative marine area column being correct but the doubling still present in the total area protected column. Therefore, if care is not taken to incorporate the correct column in the dataset, potential still exists for users of the raw database figures to double count one of the largest marine protected areas on earth.

\section{Reporting on progress towards conservation targets}

The incorrect WDPA data for both Tonga and Kiribati have propagated through regional and international reports quantifying the achievement of MPA targets. For example, both the regional report "State of Conservation in Oceania" [12] and a presentation by representatives of the Convention on Biological Diversity (CBD) 1 report Tonga's total MPA coverage as $9.4 \%$ $\left(8457 \mathrm{~km}^{2}\right)$ of their territorial waters, or $1.51 \%$ of their total marine area and highlight it as a success case for achieving the Aichi CBD Target 11 . In reality Tonga's current total MPA coverage is closer to $0.1 \%$ of its territorial waters and $0.014 \%$ of its total marine area. The same CBD presentation reports Kiribati as having reached $22 \%$ coverage of their total marine area. As the actual figure is $11 \%$ this overestimate is likely due to the issues highlighted with the WDPA dataset in Section 3. In addition, there are various international reports citing the WDPA data for global targets of marine conservation and there is a high risk that these reports could be citing incorrect figures for the South Pacific [5-8].

\section{Conclusions}

This article documents how large misrepresentations of the MPA coverage of two Pacific island countries have occurred in the WDPA and thus their contributions to achieving the Aichi 11 CBD target. This has led to overestimates of Tonga's contribution to marine protected area coverage targets by two orders of magnitude and Kiribati's by double. Large-scale misrepresentations significantly interfere with measuring progress towards marine conservation and while errors within the WDPA framework were highlighted five years ago in Visconti et al. [11], problems remain. While Visconti et al. [11] pointed to possible underestimates in protected area coverage due to the omission of community managed areas, this article points to gross overestimates. These are of greater concern, given both that conservation efforts may cease if targets appear to be met and that governments and management may aim to replicate reported successes.

The misrepresentations discussed in this article from the South Pacific are likely symptomatic of larger-scale issues regarding both balancing responsibility between government and the WDPA as well as the priority countries accord, or are able to accord, in achieving accuracy and 
accountability in this matter. It is clear that both the HCA and PIPA misrepresentations have been known for some time [13-15], but it is also evident that the responsibility for rectification has likely fallen between the perceived roles of government and the WDPA. Misrepresentations may also be driven by failures in procedure, including lack of clarity for how to make changes, lack of specified roles and lack of funding or technical capacity. Given the potential for embarrassment on the international stage, malignant issues such as either intentional misrepresentation or low initiative to highlight known problems could also exist. There is therefore a clear need to develop both greater clarity and accountability in the process of updating protected area coverage.

While systems for the updating and removal of protected areas from the WDPA do exist, any system in place from which the HCA and PIPA entries areas are not subject to removal clearly needs refining. The first priority should be to clarify the roles and responsibilities of both governments and the WDPA in the reporting and updating process. Governments should also be subject to a greater degree of external accountability from the WDPA or other external bodies. Quality control and data checking within the WDPA could also focus on large protected areas first, as these carry the greatest relative analytical weight. For regional analyses it should also not be prohibitively time consuming for the data user to check for double entries.

The WDPA data forms the backbone of reporting on global marine conservation targets and therefore it is important for countries, no matter how small, to ensure accurate and regular assessment of the status of national marine protected areas and at least annual reporting to the WDPA. This high degree of accountability will be vital for a region that is increasingly claiming moral high ground in ocean stewardship (e.g. Pohnpei Ocean Statement: A Course To Sustainability. Declaration of the Pacific Island Forum Leaders, Pohnpei, FMS). Countries and other data users are encouraged to ensure correct figures are regularly updated in the national and WDPA databases and to carefully read the WDPA guidelines [4] before using their raw data.

Finally, this assessment gives rise to some concerns. Aichi target 11 would at first glance be one of the more easily measurable of the 20 targets. However, given the complications revealed in monitoring even the geographical extent of MPAs, serious concerns may arise relating to whether it will be possible to record their effectiveness and equitability. Potential solutions to assessing effectiveness are being developed, but given the concerns that protected area coverage may not be the best indicator or approach to achieving environmental sustainability [3] more serious consideration is needed of the remaining 19 targets.

\section{Funding}

This research did not receive any specific grants from funding agencies in the public, commercial, or not-for-profit sectors. The first author was supported by an Australian PhD scholarship during writing.

\section{Conflict of interest}

None. 


\section{References}

[1] CBD, Quick guides to the Aichi Biodiversity Targets. Version 2. 02/2013, 2013.

[2] UNEP/CBD/COP/DEC/VII/5, Decision adopted by the conference of the parties to the convention on biological diversity at its seventh meeting. Conference of the Parties to the Convention on Biological Diversity, 2004.

[3] R.L. Pressey, R. Weeks, G.G. Gurney, From displacement activities to evidence-in- formed decisions in conservation, Biol. Conserv. 212 (2017) 337-348.

[4] UNEP-WCMC. World Database on Protected Areas User Manual 1.5. UNEP-WCMC: Cambridge, UK, 2017. Available at: 〈http://wcmc.io/WDPA〉 Manual.

[5] L. Burke, K. Reytar, M. Spalding, A. Perry, Reefs at Risk Revisited, World Resources Institute, Washington, DC, 2011.

[6] M. Spalding, et al Attaining Aichi Target 11: How well are marine ecosystem services covered by protected areas? World Parks Congress, Sydney, 2014.

[7] H. Thomas, B. MacSharry, L. Morgan, N. Kingston, R. Moffitt, D. Stanwell-Smith, L. Wood, Evaluating official marine protected area coverage for Aichi Target 11: appraising the data and methods that define our progress, Aquat. Conserv.: Mar. Freshw. Ecosyst. 24 (2014) 8-23.

[8] L. Wood, L. Fish, J. Laughren, D. Pauly, Assessing progress towards global marine protection targets: shortfalls in information and action, Fauna Flora Int., Oryx 42 (3) (2008) 340-351.

[9] A. Rife, B. Erisman, A. Sanchez, O. Aburto-Oropeza, When good intentions are not enough... Insights on networks of "paper park" marine protected areas, Conserv. Lett. 6 (2013) 200-212.

[10] M. Mascia, S. Pailler, Protected area downgrading, downsizing, and degazettment (PADDD) and its conservation implications, Conserv. Lett. 4 (2011) 9-20.

[11] P. Visconti, M. Marco, J. Alvarez-Romero, S. Januchowski-hartley, R. Pressey, R. Weeks, C. Rondini, Effects of errors and gaps in spatial data sets on assessment of conservation progress, Conservation Biol. 27 (5) (2013) 1000-1010.

[12] SPREP, State of Conservation in Oceania: regional report 2013. Apia, Samoa: SPREP, 2016, 2016.

[13] G. Baines, P. Hunnam, M. Rivers, B. Watson, South Pacific Biodiversity Conservation Program (SPBSP) Terminal Evaluation Report. United Nations Development Program project number RAS/91/G31/E/1G/99, 2002. 
[14] H. Govan et al. Status and potential of locally-managed marine areas in the South Pacific: meeting nature conservation and sustainable livelihood targets through wide-spread implementation of LMMAs. SPREP/WWF/WorldFish-Reefbase/CRISP. 95pp + 5 annexes.

[15] H. Govan, Area-Based Management Tools for Coastal Resources in Fiji, Kiribati, Solomon Islands, Tonga And Vanuatu. Volume 1: Status, capacity and prospects for collaborative resource management. Volume 2: Country reports. Report for the Marine and Coastal Biodiversity Management in Pacific Island Countries (MACBIO) project, Suva, Fiji Islands, 2015. 